\title{
IMPAKT Meeting 2012 - Latest Updates in Breast Cancer Translational Research
}

\author{
Robert Armbrust $^{\mathrm{a} *}$ Ruth Exner ${ }^{\mathrm{b} *}$ Sophie Doisneau-Sixou ${ }^{\mathrm{a}, \mathrm{c}}$ \\ aBrustzentrum, Universitätsfrauenklinik, Klinikum der Ludwig-Maximilians-Universität, Munich, Germany \\ ${ }^{\mathrm{b}}$ Medizinische Universität Wien, Klinik für Chirurgie, Vienna, Austria \\ 'Université Paul Sabatier Toulouse III, Toulouse, France
}

The $4^{\text {th }}$ IMPAKT (IMProving cAre and Knowledge through Translational research) Breast Cancer Conference, held in Brussels on May 2-5, 2012, gathered nearly 600 participants from countries all over the world, re-confirming itself as one of the leading events on translational research in breast cancer. Organized by the Breast International Group (BIG) and the European Society of Medical Oncology (ESMO), it was chaired by Sherene Loi and Fabrice André. This year's meeting was mainly focused on improving clinicians' understanding of the rapidly growing body of knowledge about new molecular pathways, biomarkers, drugs, and genomic technologies while updating their skills to integrate and use such new technologies. Also, for the first time in the meeting's history, there was a collaboration with the European Society of Pathology. Another main focus was to address young medical oncologists by offering a pre-conference training course.

\section{Pre-IMPAKT Training Course}

This course offered a broad teaching program in current breast cancer research as well as giving the participants the opportunity to network and interact with faculty members. Therefore well-established experts provided an educational program covering important fields of ongoing research.

A total of 71 selected young medical oncologists and researchers participated in the course that started the day before the main meeting and that lead over to the opening keynote lecture. Sherene Loi, Belgium, and Fabrice André, France, warmly welcomed the participants with a social lunch followed by a presentation about career planning in translational research. The first invited speaker was Lewis Cantley, USA who impressively illustrated his findings on the PI3K/

*The first two authors contributed equally to this report.

\section{KARGER}

Fax +497614520714

Information@Karger.de

www.karger.com
(C) 2012 S. Karger GmbH, Freiburg

Accessible online at:

www.karger.com/brc
AKT/mTOR pathway in breast cancer as well as the role of possible targeting agents. He showed that inhibition of this pathway could significantly improve the outcome in cancer treatment. Afterwards Bryan Hennesy, Ireland, suggested that PI3K pathway aberrations are associated with resistance to trastuzumab and cytotoxic therapy and that a combination with the mTOR inhibitor everolimus could improve the outcome in trastuzumab resistant cancer. During the second lecture concepts of drug development were presented from different points of view: Javier Cortes, Spain, showed his perspective as a clinician and was then followed by the pharma's viewpoint represented by Cornelia Quadt from Novartis, showing the development of the alpha specific PI3K inhibitor BYL719. Stefan Michiels, Belgium, presented then as a statistician very relevant possibilities of improving trial designs. Afterwards Jean-Yves Pierga, France, and Arkaitz CarracedoPerez, Spain, demonstrated updates in monitoring angiogenesis and in tumor metabolism. But the highlight was the talk of Mark J. Smyth, Australia, who presented his findings on the role of the immune system in breast cancer and how immunotherapeutic approaches could be developed in the future.

The next day, lectures provided updates on new technologies. Jorge Reis-Filho, UK, and Christine Desmedt, Belgium, introduced new approaches made possible through next-generation sequencing technologies, with some emphasis on the different possibilities of RNA sequencing, such as the detection of RNA editing and gene fusion events. Then Leanne de Koning, France, showed how kinase activity can be quantified by using Reverse Phase Protein Arrays, bringing it out as a very promising tool. Subsequently Michail Igniatidis, Belgium, promoted very interesting insights on the value of circulating tumor cells presented as potential liquid biopsy for monitoring tumor phenotype and genotype. Finally, Charles Swanton, UK, closed the training course by presenting impressively his findings on intratumor heterogeneity (ITH). 


\section{Main Meeting}

Immediately after the training course, the main IMPAKT meeting 2012 was opened featuring a remarkable list of invited speakers. Several keynote lectures concentrated on new findings on ITH, triple negative breast tumors, clinical perspectives and implications of targeting the PI3K pathway, and the value of biomarkers. The first keynote lecture was opened by Lewis Cantley, USA, who clearly demonstrated that an activated PI3K pathway, PIK3CA amplification, and PTEN loss are significantly associated with a shorter progression free survival and that pathway inhibiting agents are promising. But he also indicated that phase II trials will be needed to select patients based on biomarkers (e.g., mutational status) due to reported sporadic response to PI3K inhibitors. Nonetheless José Baselga, USA, could show that the benefit of detecting biomarkers for decision-making in adjuvant breast cancer treatment is still questionable and that the current multigene signatures are not convincing enough so far.

During the next session Gunter von Minckwitz, Germany, reported his conclusions based on the recently published results of the neoadjuvant GEPARquinto trial. He mainly concluded that a better identification of low-risk breast cancer subtypes, which would benefit from more and better chemotherapy, is necessary. Martine Piccart, Belgium, gave then a reliable update on landmark translational research from 2010 to 2011. She provided a very distinctive overview on the quality of multigene signatures as possible response predictors and concluded that none of the important trials of the last 3 years identified reliable biomarkers. For example she demonstrated that the so called A-Score (combination of immune signature, stroma signature, and TOP2A signature) could be a predictor for an anthracyclin benefit in estrogen receptor (ER) negative breast cancer [Desmedt et al., J Clin Oncol 2011].

In the afternoon session Tak Mak, Canada, shed light on mechanisms of cancer cells to protect against metabolic stress (hypoxia, oxidative stress, glucose deprivation) via specific pathways, driven in particular by the carnitine palmitoyl transferase1C [Zaugg et al., Genes Dev 2011]. Michael Pollak, Canada, pointed out that insulin is of greater importance to tumor cell metabolism than glucose itself and explained the protective effect of metformin and phenformin via downregulation of mTOR, through an energy stress and AMPK signaling, leading to apoptosis and inhibition of proliferation. Alastair Thompson, UK, emphasized the importance of the tumor surrounding stroma and of the use of stromal markers to predict clinical outcome in triple negative breast cancer [Witkiewicz et al., Cell Cycle 2012].

The next session on biomarker and drug development was opened by Nicolas Turner, UK, who clearly explained the alterations of fibroblast growth factors pathways in breast cancers, distinguishing FGFR-1 (25\% luminal B) and FGFR-2 (rare) amplifications. Basal-like breast cancers, by expressing FGF2, demonstrate sensitivity to FGFR inhibitors, identifying a potential novel therapeutic approach [Sharpe et al., Clin Cancer Res 2011]. Fabrice André, France, recapitulated the limits of clinical trials on rare populations of patients and developed non selective and selective FGFR inhibitors, stressing the perspective of combination with endocrine therapy.

The second day of the meeting, the morning session was dedicated to ITH and the clinical perspectives with two keynote lectures. Charles Swanton, UK, first presented a clearcut picture of challenges and opportunities offered by improved knowledge of ITH. He explained the relevance of ITH and cancer branched evolution revealed by multiregion sequencing [Gerlinger et al., N Engl J Med 2012], as ITH may foster tumor adaptation, poorer outcome, and therapeutic failure through Darwinian selection. He emphasized the necessity in the future to track tumor subclonal architecture dynamics. Jonas Bergh, Sweden, gave then a nice update on the clinical perspectives of ITH, outlining the limits of the actual ER assessment in the primary tumor obviously already exhibiting heterogeneity. The conclusions from meta-analyses from 100,000 patients in adjuvant settings [EBCTCG, Lancet 2012] are that chemotherapy may add value for all patients, even ER positive patients, but that we are still lacking tumor gene expression markers (prospective data from MINDACT, TAILORx) or quantitative immunohistochemistry that might help to predict risk, chemosensitivity, or both. Very logically, Peter Campbell, UK, followed then by presenting how the next generation sequencing (NGS) can decipher ITH, tracking the evolution of cancer genomes in time and giving insights in the sub-clonal diversification: a dominant initial subclone is likely to trigger diagnosis and, besides the numerous mutations that each clone carries, implies long-lived lineage of cells passively accumulating mutations. Christoph Klein, Germany, discussed then the major extent of selection and adaptation outside the primary tumor of disseminated tumor cells. Genome and transcriptome changes may be by far more dynamic than suggested from cell line models. Matthew Ellis, USA, recapitulated then how by sequencing somatic variants sites at high depth, models of therapeutic heterogeneity can be built with implications for these numerous actionable targets.

In the afternoon, triple negative breast cancer (TNBC) segmentation was widely presented and discussed with the very nice talk from Jorge Reis-Filho, UK. He emphasized the populations of TNBC that are not basal-like (claudin-low, molecular apocrine) and the basal-like that are not TNBC (HER2 positive), as TNBC are heterogeneous in their response to chemotherapy. He concluded that transcriptomic subtyping should have prognostic and new drug development implications. Samuel Aparicio, Canada, reaffirmed how new insights in orphan subtypes are essential to avoid exclusion diagnosis and presented a fascinating work on the clonal and mutational evolution spectrum of TNBC, using high-throughput RNA sequencing (RNA-seq) [Shah et al., Nature 2012]. 3 key concepts emerge: clonal frequency, clonal genotype, 
and clonal lineage, with the identification for around $20 \%$ TNBC of specific actionable mutations that cannot not be characterized through cell line models.

A specific session was dedicated to targeting the host and microenvironment in TNBC. Mark J. Smyth, Australia, presented the developments of immunotherapy, e.g., anti-CD73. Stefan Scherer from Roche, Switzerland, nicely overviewed the lessons learnt from biomarker development in industry and outlined the limitations of sample collection and the need for an integrative biomarker strategy including large global trials, e.g., VEGF-A as bevacizumab marker.

A last session introduced innovative targeted drugs in TNBC. Thomas Helleday, Sweden, after recapitulating the PARP inhibitor action presented the targeting of Chk1 in p53-deficient TNBC [Ma et al., J Clin Invest 2012] and Andrea Alimonti, Switzerland, presented the crucial role of PTEN. Eric Winer, from Dana-Farber, USA, completed this session by recapitulating how to optimize future drug development for each TNBC subtype, basically taking into account the heterogeneity of the disease, developing neo-adjuvant settings and bringing together clinic trialists and laboratory scientists.

A highlight of the congress was the gene array session chaired by Fraser Symmans, USA, and Eric Winer, USA, on the last day of the meeting. Christos Sotiriou, Belgium, presented an overview of first and new generation gene signatures [Haibe-Kains et al., J Natl Cancer Inst 2012; Curtis et al., Nature 2012], their prognostic value and possible ability to predict drug sensitivity. He recently showed the association between neoadjuvant chemotherapy response and gene expression modules describing important biologic processes, such as the immune module that added to clinicopathologic characteristics substantially increases predictive accuracy for pCR in the HER2-pos subtype [Ignatiadis M. et al., J Clin Oncol 2012]. Fraser Symmans, USA, explained then the feasibility of gene arrays in clinical practice mentioning the challenge of adequate pre-analytical conditions, e.g. optimal preservation of the tumor with RNALater ${ }^{\circledR}$. Dan Hayes, USA, defined the important criteria for implementation of whole genome arrays in clinical practice according to the ASCO tumor marker guidelines panel.
In the pathology research session, an excellent overview of new markers in pathology and gene profiles was given by Carsten Denkert, Germany, including the EndoPredict ${ }^{\circledR}$ test [Filipits M. et al., Clin Cancer Res 2011], which identifies patients with excellent prognosis in ER positive Her2 negative breast cancers. He also presented a study showing frequently expressed Mucin 1 especially in hormone receptor positive tumors to be predictive for pathological complete response (pCR) in the GeparTrio trial. Guiseppe Viale, Italy, presented strategies for improving inter- and intra-laboratory concordance and suggested a registry for gene profiles to accumulate data from patients out of clinical trials. He stressed the level $1 \mathrm{~b}$ evidence of gene signatures and their impact on prognosis and not prediction and the need of further results, e.g. from the Mindact trial.

In the final guidelines session, Charles Swanton, UK, explained the benefit of gene signatures compared to standard immunohistochemical diagnostic in daily practice like differentiation between luminal A and luminal B in ER positive disease. The cut-off point of proliferation index KI 67 at 14\% was discussed and reaffirmed by Fabrice André, France, to be a value from a rock curve. Jorge Reis-Filho, UK, concluded by giving an excellent interactive lecture about the definition and comparison of molecular subclasses (using immunohistochemistry, luminal A/B-KI67 or PAM50) and their impact on therapy.

Altogether, the IMPAKT meeting was a scientific success, including satellite symposia (personalized medicine, biomarkers of endocrine therapy resistance, and Herceptin subcutaneous), posters (with 20 travel grants awarded) and best abstract sessions to provide the right environment for young professionals to network with and access the knowledge of their senior counterparts. The 2013 IMPAKT meeting in Brussels should not be missed. It will be chaired by Peter Dubsky, Austria, and Andrew Tutt, UK, with once again an exciting scientific program (www.impakt.org). New and clinically relevant translational research findings will also be discussed at the 5th COMBATing Breast Cancer Meeting (Chairs: Nadia Harbeck, Michael Gnant, Achim Rody) from Nov 23 to 24, 2012, in Munich, Germany (www.combating.de). 